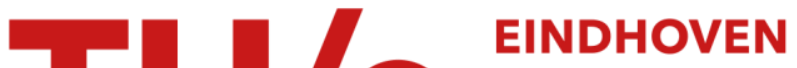 \\ UNIVERSITY OF \\ TECHNOLOGY
}

\section{Stability parameter identification for a centrifugal compression system}

\section{Citation for published version (APA):}

Helvoirt, van, J., Jager, de, A. G., Steinbuch, M., \& Smeulers, J. P. M. (2004). Stability parameter identification for a centrifugal compression system. In Proceedings of the 43rd Conference on Decision and Control (CDC 2004), 14-17 December, 2004, Atlantis, Bahamas (pp. 3400-3405). Institute of Electrical and Electronics Engineers. https://doi.org/10.1109/CDC.2004.1429234

DOI:

10.1109/CDC.2004.1429234

Document status and date:

Published: 01/01/2004

\section{Document Version:}

Publisher's PDF, also known as Version of Record (includes final page, issue and volume numbers)

\section{Please check the document version of this publication:}

- A submitted manuscript is the version of the article upon submission and before peer-review. There can be important differences between the submitted version and the official published version of record. People interested in the research are advised to contact the author for the final version of the publication, or visit the $\mathrm{DOI}$ to the publisher's website.

- The final author version and the galley proof are versions of the publication after peer review.

- The final published version features the final layout of the paper including the volume, issue and page numbers.

Link to publication

\section{General rights}

Copyright and moral rights for the publications made accessible in the public portal are retained by the authors and/or other copyright owners and it is a condition of accessing publications that users recognise and abide by the legal requirements associated with these rights.

- Users may download and print one copy of any publication from the public portal for the purpose of private study or research.

- You may not further distribute the material or use it for any profit-making activity or commercial gain

- You may freely distribute the URL identifying the publication in the public portal.

If the publication is distributed under the terms of Article $25 \mathrm{fa}$ of the Dutch Copyright Act, indicated by the "Taverne" license above, please follow below link for the End User Agreement:

www.tue.nl/taverne

Take down policy

If you believe that this document breaches copyright please contact us at:

openaccess@tue.nl

providing details and we will investigate your claim. 


\title{
Stability Parameter Identification for a Centrifugal Compression System
}

\author{
Jan van Helvoirt, ${ }^{*}{ }^{\ddagger}$ Bram de Jager, ${ }^{*}$ Maarten Steinbuch, ${ }^{*}$ Jan Smeulers, ${ }^{\ddagger}$ \\ *Technische Universiteit Eindhoven \\ Dep. Mechanical Engineering \\ Dynamics \& Control Technology \\ P.O. Box 513, $5600 \mathrm{MB}$ \\ ‡TNO TPD \\ Flow \& Structural Dynamics \\ P.O. Box 155, 2600 AD \\ Delft, The Netherlands
}

Eindhoven, The Netherlands

\begin{abstract}
This paper presents the application of a lumped parameter model to describe the dynamic behavior of a centrifugal compression system including surge. The response of the model is compared with experimental surge measurements from an industrial single stage compressor test rig. A parametric analysis of the model reveals the large influence of the stability parameter on the transient response. However, a good value for this parameter is not easily obtained from surge data. Therefore, an identification method is proposed to uniquely determine the stability parameter that is based on an approximate realization algorithm, making use of the fact that the step response of the system has the characteristics of a first order system. Simulation results show that this method can provide an estimate for the stability parameter of a centrifugal compression system.
\end{abstract}

\section{INTRODUCTION}

Compressor surge is a large amplitude oscillation of the total annulus averaged mass flow through the compressor. This unstable axisymmetric flow pattern and the associated pressure rise fluctuations limit the stable operating range of the compressor towards low mass flows. Surge not only reduces performance and efficiency, it can also damage the compressor due to large mechanical and thermal loads.

Over the last decade, much work has been done on the analysis and modeling of compressor instabilities. In particular we mention the development of the nonlinear Greitzer model of transient compression system behavior [1]. This model exploited the analogy between surge oscillations and a Helmholtz resonator. To this day it is the most widely used dynamic model in the field.

The Greitzer lumped parameter model contains an important parameter that characterizes the dynamic response of the compression system. This stability parameter is usually determined from geometric data of the system. But for centrifugal compressors this is not straightforward due to the complex geometry of a centrifugal compression system [2], [3]. The authors solved this problem by tuning the parameters in such a way that good agreements between the model predictions and actual surge measurements were obtained.

Corresponding author is J. van Helvoirt, E-mail: j.v.helvoirt@tue.nl
This paper presents the application of the Greitzer model to an experimental compression system. We will adjust the model to account for the effect of the return piping, a feature of the setup usually not encountered in industrial compression systems. The model predictions will then be compared with data from surge experiments and we will carry out a parametric analysis of the developed surge model. We will reveal the existence of a non-uniqueness when the stability parameter is tuned to match surge data.

The main contribution of this paper is a method to determine a realistic stability parameter for the Greitzer model of a centrifugal compression system. The stability parameter is determined from a linear time-invariant (LTI) system that approximates the relevant dynamics of the actual system. This LTI system is extracted from measured step response data, using a modified approximated realization algorithm [4]. Simulation results are presented to show that the proposed method is suitable for estimating the stability parameter of the studied compression system.

\section{COMPRESSION SYSTEM MODELING}

This section deals with the development of a dynamic model for an industrial compressor test rig. After introducing the actual compression system, the nonlinear lumped parameter model will be presented. Despite some minor modifications, this model will be similar to the original Greitzer model.

\section{A. Experimental setup}

Experiments were done on an single stage compressor test rig that is normally used to test industrial compressors. The centrifugal compressor is driven by an electric motor that is connected to the shaft through a gearbox. The rotational speed can be varied between 8,000 and 16,000 rpm. Surge experiments are performed for rotational speeds of $9,000,11,000,13,000$, and 15,000 rpm.

The compressor operates in a closed circuit that makes it possible to use different pure gases or gas mixtures. Furthermore, this configuration allows for varying the average pressure in the system between 1 and 15 bar. All results 
TABLE I

PARAMETERS OF THE COMPRESSION SYSTEM

\begin{tabular}{|c|l|c|l|}
\hline Element & Parameter & Value & Unit \\
\hline \hline \multirow{2}{*}{ Impeller } & number of blades & 17 & - \\
& inlet diam. at casing $d_{i, c}$ & 0.1592 & $\mathrm{~m}$ \\
& inlet diam. at hub $d_{i, h}$ & 0.1450 & $\mathrm{~m}$ \\
& exit diameter $d_{e}$ & 0.2840 & $\mathrm{~m}$ \\
& exit width & 0.0043 & $\mathrm{~m}$ \\
Vaned diffuser & number of vanes & 10 & - \\
& inlet diameter & 0.2870 & $\mathrm{~m}$ \\
& outlet diameter & 0.4409 & $\mathrm{~m}$ \\
& width & 0.0036 & $\mathrm{~m}$ \\
& piping volume $V_{1}$ & 3.0693 & $\mathrm{~m}^{3}$ \\
& discharge volume $V_{2}$ & 0.3204 & $\mathrm{~m}^{3}$ \\
& compressor duct area $A_{c}$ & 0.0034 & $\mathrm{~m}^{2}$ \\
& compressor duct length $L_{c}$ & 0.1970 & $\mathrm{~m}$ \\
\hline
\end{tabular}

presented in this paper were obtained from experiments with $N_{2}$ gas $\left(28.0134 \cdot 10^{-3} \mathrm{~kg} / \mathrm{mol}\right)$ and an average suction pressure of 10 bar.

Throttling of the compressor is done by means of a butterfly valve. A parallel control valve is used for more precise adjustments of the mass flow rate. The return piping contains a measurement section with a flow straightener, an orifice flow meter, and a gas cooler. With the combination of the throttle, return piping, and cooler, the conditions of the gas at the compressor inlet, are kept approximately constant. Some characteristics of the installation are summarized in Table I. We remark that $V_{2}$ represents the combined volume of the discharge pipe and the compressor volute.

The compression installation is equipped with numerous temperature probes and static pressure transducers to determine the steady-state performance of the compressor. A dedicated data-acquisition and control system is used for operating the installation, converting and recording sensor outputs, and for online monitoring.

Additional pressure transducers were installed in the suction and discharge pipes to measure the pressure rise fluctuations during surge. For data-acquisition a SIGLAB system with anti-aliasing filters and A/D convertors was used. All dynamic pressure signals were measured at a sampling rate of $5.12 \mathrm{kHz}$.

\section{B. Model equations}

To describe the dynamic behavior of the compression system we used a lumped parameter model, following the geometry of Fig. 1. The derivation of the proposed model was analogous to [1], yielding

$$
\begin{aligned}
\frac{d \phi_{c}}{d \tilde{t}} & =B\left(\psi_{c}\left(\phi_{c}\right)-\psi\right) \\
\frac{d \psi}{d \tilde{t}} & =\frac{F}{B}\left(\phi_{c}-\phi_{l}\left(\psi, u_{l}\right)-\phi_{s}\left(\psi, u_{s}\right)\right)
\end{aligned}
$$

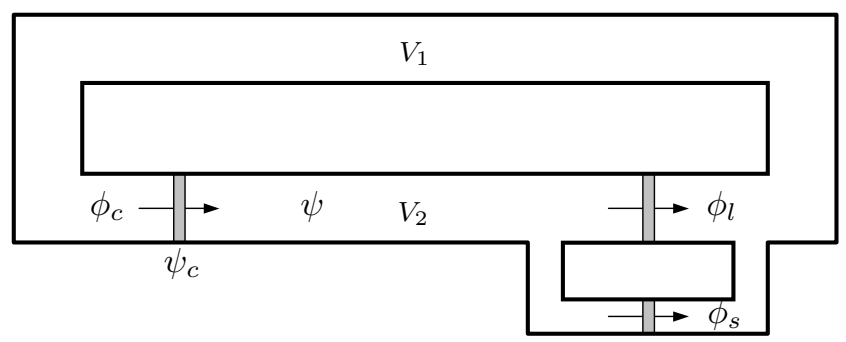

Fig. 1. Lumped parameter model.

where $\phi_{c}$ and $\psi$ are defined as

$$
\phi_{c}=\frac{\dot{m}_{c}}{\rho_{1} A_{c} U} \text { and } \psi=\frac{\Delta p}{\frac{1}{2} \rho_{1} U^{2}}
$$

and time is scaled with the Helmholtz frequency $\omega_{H}$

$$
\tilde{t}=t \cdot \omega_{H}=t \cdot a_{2} \sqrt{\frac{A_{c}}{V_{2} L_{c}}}
$$

The Greitzer stability parameter $B$ is given by

$$
B=\frac{U}{2 \omega_{H} L_{c}}=\frac{U}{2 a_{2}} \sqrt{\frac{V_{2}}{A_{c} L_{c}}}
$$

This parameter can be regarded as the ratio between the resistive and inertial forces within the system [1]. Stated differently, large values of $B$ imply that the time scale of the surge oscillation is set by a balance between resistive and restoring forces. Here, the restoring forces are associated with the potential energy of the compressed fluid in the plenum. For lower values of $B$ the time scale of the motions is set basically by a balance between the inertial and restoring forces.

The difference with the original Greitzer model is that in Fig. 1 the return piping couples the throttle exit to the compressor inlet. Therefore, the principle of mass conservation was applied to both volumes $V_{1}$ and $V_{2}$ separately. Because the total amount of mass in the system is constant we then combined the two resulting mass balances. This yielded a single differential equation for the pressure difference $\Delta p$. The effect of the coupling is now accounted for in (2) by the parameter $F$

$$
F=1+\frac{Z_{1} T_{1} V_{2}}{Z_{2} T_{2} V_{1}}
$$

that was obtained from the aerodynamic scaling of the combined mass balance. When $V_{1}$ becomes infinitely large, the original Greitzer model is obtained.

Similar to [5], the dimensionless compressor characteristic $\psi_{c}\left(\phi_{c}\right)$ is approximated by a cubic polynomial in $\phi_{c}$. The point $\psi_{c}(0)$ is determined analytically according to [6]. The resulting curves are shown in Fig. 2. For subsonic flow conditions, the dimensionless throttle characteristics can be approximated by

$$
\phi_{i}\left(\psi, u_{i}\right)=c_{i} u_{i} \sqrt{\psi}, \quad i=l, s
$$

The throttle parameters $c_{l}$ and $c_{s}$ were determined from valve data, yielding $c_{l}=0.56$ and $c_{s}=0.017$. 


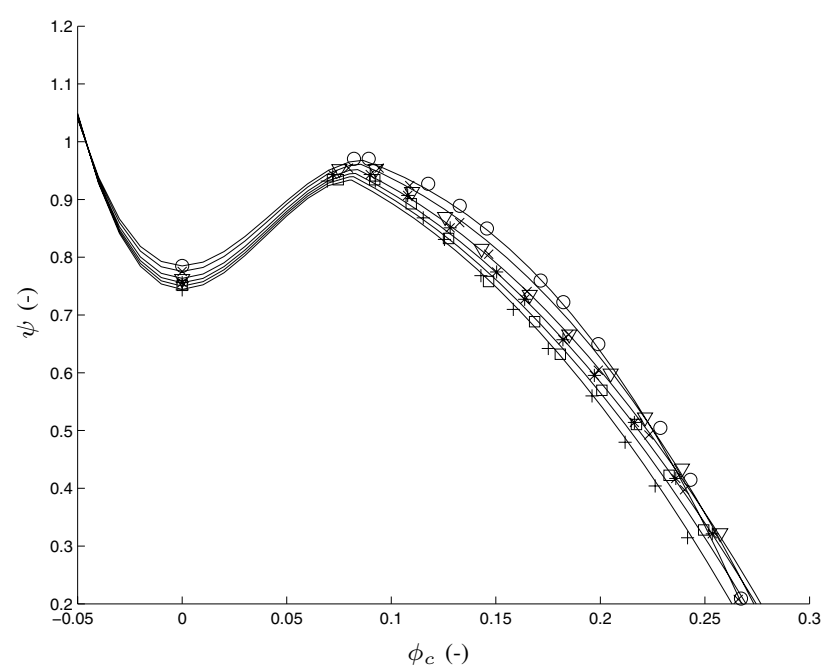

Fig. 2. Dimensionless compressor curves for $N=7,829 \mathrm{rpm}(+)$; $8,961 \mathrm{rpm}(\square) ; 10,091 \mathrm{rpm}(*) ; 11,510 \mathrm{rpm}(\nabla) ; 13,410 \mathrm{rpm}(\times)$; and 15, $061 \mathrm{rpm}(\mathrm{\circ})$

TABLE II

ADDITIONAL NOMENCLATURE

\begin{tabular}{|c|c|c|}
\hline Symbol & Description & Unit \\
\hline$a$ & speed of sound & $\mathrm{m} / \mathrm{s}$ \\
\hline$A$ & area & $\mathrm{m}^{2}$ \\
\hline$c_{p}$ & specific heat at constant pressure & $\mathrm{J} / \mathrm{kg} \cdot \mathrm{K}$ \\
\hline$d$ & diameter & $\mathrm{m}$ \\
\hline$f_{s}$ & sample frequency & $\mathrm{Hz}$ \\
\hline$L$ & length & $\mathrm{m}$ \\
\hline$\dot{m}$ & mass flow rate & $\mathrm{kg} / \mathrm{s}$ \\
\hline$N$ & rotational speed & $\mathrm{rpm}$ \\
\hline$p$ & pressure & $\mathrm{Pa}$ \\
\hline$t$ & time & $\mathrm{s}$ \\
\hline$\tilde{t}$ & scaled time & - \\
\hline$T$ & temperature & K \\
\hline$u$ & normalized valve position & \\
\hline$U$ & impeller tip velocity & $\mathrm{m} / \mathrm{s}$ \\
\hline$Z$ & compressibility factor & \\
\hline$\gamma$ & specific heat ratio & - \\
\hline$\Delta p$ & pressure difference $p_{2}-p_{1}$ & $\mathrm{~Pa}$ \\
\hline$\rho$ & density & $\mathrm{kg} / \mathrm{m}^{3}$ \\
\hline$\phi$ & flow coefficient & - \\
\hline$\phi_{c, s}$ & flow coefficient at surge point & - \\
\hline$\psi$ & pressure coefficient & - \\
\hline Subscript & Description & \\
\hline 0 & nominal value & \\
\hline 1 & piping, suction side & \\
\hline 2 & plenum, discharge side & \\
\hline$c$ & compressor & \\
\hline$l$ & large throttle & \\
\hline$s$ & small throttle & \\
\hline $\begin{array}{c}\text { Supercript } \\
\sim\end{array}$ & $\begin{array}{l}\text { Description } \\
\text { estimation } \\
\text { perturbation }\end{array}$ & \\
\hline
\end{tabular}
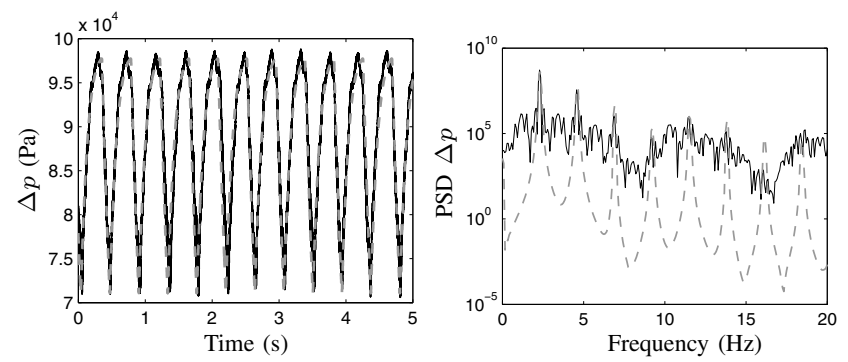

Fig. 3. Simulation (- -) and experimental (-) results for $N=9000 \mathrm{rpm}$ and $B=4.16$.

\section{MODEL ANALYSIS}

This section focuses on the analysis and validation of the compression system model. We make a comparison between the simulation results and actual surge measurements. We then show that the model parameters cannot be uniquely defined on the basis of surge measurements alone. Finally we illustrate the effect of this ambiguity on the local dynamics of the linearized compression system model.

\section{A. Surge simulations}

The model was validated by simulating all the experimental operating conditions and comparing the results with experimental data. The initial values for the geometric parameters of the model can be found in Table I. Values for $a, Z$, and $\rho$ were obtained from gas property tables.

Results obtained with the presented model are shown in Fig. 3 for one operating condition. The plots show that both the pressure rise fluctuations and the frequency of the surge oscillations were predicted well. Next to the surge frequency also the first five higher order harmonics of the oscillation were accurately predicted. Similar results were obtained for other operating conditions.

\section{B. Parametric analysis}

The effect of the value for $B$ on the dynamic behavior during surge will now be discussed in greater detail. For our initial simulations we have used a rather small value for $L_{c}$ (yielding a large $B$ ), similar to [6, p. 33]. In literature normally a larger value is used, based on the length between the compressor inlet and a large plenum volume [2], [7]. The problem of the investigated test rig is that the duct and volume cannot be distinguished clearly within the system. This difficulty is common for industrial compression systems, in contrast to dedicated laboratory setups.

Despite the uncertain physical interpretation, we increased $L_{c}$ from 0.197 to 1.8 and $3 \mathrm{~m}$. With the resulting values of $B$, new simulations were carried out. The remaining parameters of the model were kept the same during these simulations. The resulting pressure rise data in Fig. 4 and 5 show that the predicted surge frequencies were still in 

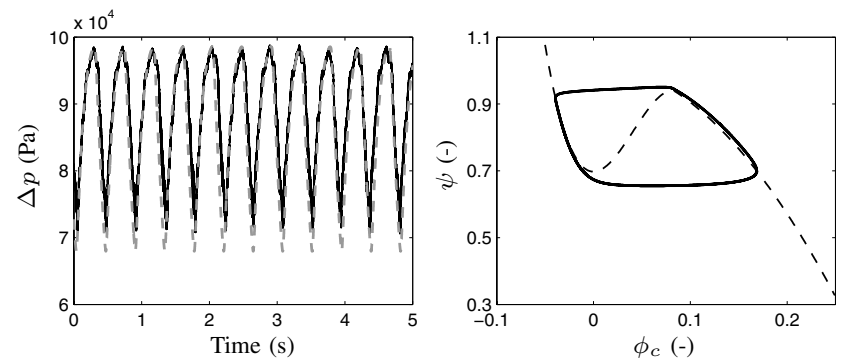

Fig. 4. Results with $L_{c}$ increased from 0.1970 to $1.8 \mathrm{~m}, N=9000 \mathrm{rpm}$ and $B=1.38$ : simulated (- - and measured (-) pressure rise (left figure); surge oscillations (-) and steady-state (- -) compressor curve (right figure).
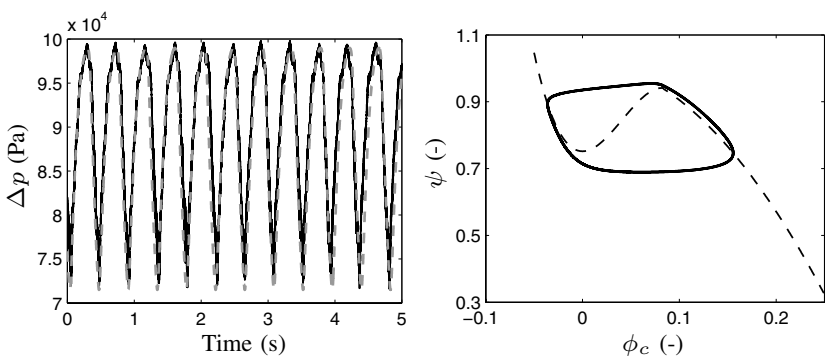

Fig. 5. Results with $L_{c}$ increased from 0.1970 to $3 \mathrm{~m}, N=9000 \mathrm{rpm}$ and $B=1.07$ : simulated (- - ) and measured (-) pressure rise (left figure); surge oscillations (-) and steady-state (- -) compressor curve (right figure).

relatively good agreement with the measurements, despite the different $B$ parameters.

Furthermore, the results showed that the minimum pressures of the simulated surge cycles were lower than the measured values. However, the calculation of $\psi_{c}(0)$ (see the Appendix) was not believed to be very accurate, given the complexity of the flow patterns in the impeller and diffuser at zero mass flow. Therefore it seemed justifiable to shift the valley point of the compressor characteristic, in order to reduce the difference between simulated and measured surge oscillation amplitudes. We verified that shifting the valley point upwards (max. 8\%) again resulted in good agreement between simulations and measurements. Note that the plots of the limit cycle oscillations in the $\phi_{c}-\psi$ plane do not contain any experimental data, since no instantaneous mass flow measurements were available.

So far we have showed that using different values for the stability parameter $B$ barely influenced the agreement between predictions and measurements of both surge amplitude and frequency. We will now take a closer look at the effect of $L_{c}$ (i.e., the value of $B$ ) on the local dynamic behavior. Therefore, we linearize (1)-(2) around $\left(\phi_{c 0}, \psi_{0}, u_{l 0}, u_{s 0}\right)$. For the resulting linear system we have plotted the location of the system poles ${ }^{1}$ as a function of $B$. From the results shown in Fig. 6 it becomes clear that

\footnotetext{
${ }^{1}$ Roots of the characteristic equation $\operatorname{det}(s \mathbf{I}-\mathbf{A})=0$
}
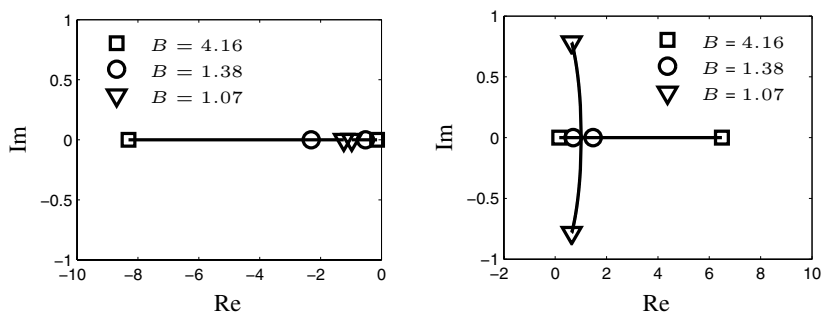

Fig. 6. Root locus for the linear compression system with varying $B$ at $N=9000 \mathrm{rpm}: \phi_{c 0}=1.1 \phi_{c, s}$ (left figure); $\phi_{c 0}=0.9 \phi_{c, s}$ (right figure).

varying $B$ has a drastic effect on the local dynamic behavior of the compression system in the unstable flow regime.

The significant change in the transient response of the compressor model (solely caused by selecting another value for $B$ ) does not affect the predictions of the surge amplitude and frequency, as shown in Fig. 4 and 5. However, the pole locations (real or complex conjugate) of the linear compressor model can have a significant impact on, for example, the design of active surge controllers [3], [8].

From the above analysis, we conclude that the $B$ parameter characterizes the dynamic behavior of the compression system. Therefore, in order to develop an accurate model for the investigation of the local dynamics during compressor surge, an accurate value for $B$ must be available. In industrial compression systems however, the determination of $B$ from the compressor geometry is not straightforward. We suggest a solution to this problem in the next section.

\section{STABILITY PARAMETER IDENTIFICATION}

A new identification method for $B$ that is based on measurements from the actual system will be proposed in this section. First we briefly discuss an approximate realization algorithm that forms the basis for the identification method. The approximate realization algorithm is chosen for its straightforward implementation and computational simplicity, while providing enough flexibility and insight in order to tune the algorithm properly. With the identification method we can resolve the ambiguity between the local dynamics and measurements of surge amplitudes and frequencies. This is confirmed through simulations. We finish by giving some additional suggestions for making the method applicable in practice and presenting the final simulation results.

\section{A. Approximate realization}

A straightforward way to obtain an approximate realization for a stable discrete-time LTI system is via the construction of a Hankel matrix from a finite sequence of Markov parameters. A model of appropriate order $\rho$ is then calculated from a singular value decomposition (SVD) of this Hankel matrix [9].

Since the exact Markov parameters are almost never available in practice, the realization will only be an approximation of the actual dynamics. In [10] a method is 


$$
\hat{B}=\frac{F\left(1-m_{c} m_{t}\right)+\lambda^{2}+\sqrt{F^{2}\left(1-m_{c} m_{t}\right)^{2}+2 F \lambda^{2}\left(1+m_{c} m_{t}\right)+\lambda^{4}}}{2 \lambda m_{c}}
$$

suggested to obtain such an approximate realization from impulse response data. The modification suggested in [4] that allows the use of step response data, forms the basis of the identification method for the stability parameter $B$. The details of the algorithm can be found in [11, p. 25].

\section{B. Identification method}

We now return to the compression system and the problem of determining the stability parameter $B$. To solve this problem, we propose a straightforward identification method. The idea of this method is to obtain an approximated realization $(\hat{\mathbf{A}}, \hat{\mathbf{B}}, \hat{\mathbf{C}}, \hat{\mathbf{D}})$ that accurately describes the relevant compression system dynamics. When such a realization is available, the eigenvalue(s) of $\hat{\mathbf{A}}$ will approximate the eigenvalues of the system matrix for the actual system. An estimate for $B$ is then easily obtained from the analytical solution of the characteristic equation $\operatorname{det}(\lambda \mathbf{I}-\hat{\mathbf{A}})$, with $\lambda$ the corresponding eigenvalue of $\hat{\mathbf{A}}$.

So, the first step of this identification method is to obtain an approximated realization for the compression system. The previously discussed algorithm suits our needs, because the required step response sequence is relatively easy to obtain from forced response measurements on the compression system. Such measurements are done by applying a step to the control valve and measuring the resulting pressure rise over a certain period of time. Note that, when the applied perturbation is not equal to a unit step, the response should be normalized accordingly.

The size of the applied step must be large enough to result in a measurable response. However, the perturbation must remain small to stay close to the nominal operating point. Otherwise, the linear approximation for the local dynamics would no longer be valid. The choice for a suitable length $n$ of the step response mainly depends on computational limitations. A longer sequence would provide a better approximation at the cost of longer computer times. In the following subsection we will discuss these issues in somewhat greater detail.

Bode diagrams of the extended LTI system, obtained after linearizing (1)-(2) with $u_{s}$ as input, showed that the input-output behavior has the characteristics of a first order system. This "order reduction" was caused by the zero in the transfer function $H(s)=\mathbf{C}(s \mathbf{I}-\mathbf{A})^{-1} \mathbf{B}$. This zero is located close to one of the poles and therefore the linear model effectively behaved like a first order system. From this observation we concluded that a first order approximate realization should be sufficient to describe the compression system dynamics. The pole of such a first order approximate realization is equal to $\lambda$. This scalar provides an estimate for the pole of the linear system. By analyzing the root locus plots we verified that this estimate corresponds to the pole that is located closest to the imaginary axis. An estimate $\hat{B}$ for the actual stability parameter can now be calculated via (8) where

$$
\begin{aligned}
& m_{c}=\left.\frac{\partial \psi_{c}}{\partial \phi_{c}}\right|_{\phi_{c 0}} \\
& m_{t}=\left.\frac{\partial\left(\phi_{l}+\phi_{s}\right)}{\partial \psi}\right|_{\left(\psi_{0}, u_{l 0}, u_{s 0}\right)}
\end{aligned}
$$

followed from the linearization.

\section{Simulations}

The proposed identification method was evaluated by applying it to the nonlinear model (1)-(2). Simulations were carried out with the following sets of parameter values: $\phi_{c 0} / \phi_{c, s} \in\{1.1,1.25,1.5,2,2.5\}$, $\tilde{u}_{s} \in\{0.005,0.001,0.01\}, L_{c} \in\{0.197,1.8,3\}, n \in$ $\{500,1000,2000\}$, and $f_{s} \in\{1024,5120\}$.

From the results of those simulations, the following conclusions were drawn. First of all, the identification method resulted in accurate estimations of the stability parameter $B$. The order of magnitude of the estimation error, defined as $|B-\hat{B}|$, was 0.1 or less for all cases. Secondly, varying $\phi_{c 0} / \phi_{c, s}$ and $\tilde{u}_{s}$ did not lead to significant differences in the estimation error. With respect to $n$ and $f_{s}$, simulations showed that these parameters should be chosen large enough. Jointly, they should assure that the resulting step response sequence $\left\{S_{k}\right\}_{k=0}^{n}$ includes the steady-state part of the response. Finally, we concluded that the largest estimation errors were obtained from simulations with a low $B$ value. This is due to the fact that the first order approximation becomes less accurate for low $B$ values, because the two system poles are located closer together in those cases. Based on these findings, we selected the following appropriate parameter settings for further simulations: $\phi_{c 0} / \phi_{c, s}=2.5, \tilde{u}_{s}=0.01, n=2000$, and $f_{s}=1024$.

In order to obtain a more realistic simulation model, the non-ideal dynamics of the control valve should be taken into account. Therefore, the ideal step input was replaced by the step response of a dynamic filter. We used a second order filter, with an undamped natural eigenfrequency of $10 \mathrm{~Hz}$ and a dimensionless damping of 0.7 , to represent the valve dynamics. Furthermore, we added artificial measurement noise to the step response of the simulation model. For this purpose we used a white noise sequence with a variance equal to that of the experimental data.

Simulations showed that both modifications of the model drastically reduced the accuracy of the identification method. We solved this problem by performing a zerophase, low-pass filter operation on the noisy data, in order to reduce the influence of measurement noise. Moreover, 

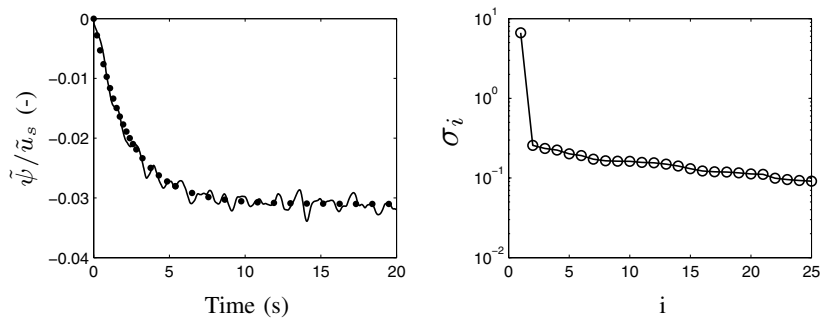

Fig. 7. Simulation results for the compression system with valve dynamics and $N=9000 \mathrm{rpm}, B=1.07, \phi_{c 0}=2.5 \phi_{c, s}, \tilde{u}_{s}=0.01, n=2000$, $f_{s}=1.024 \mathrm{kHz}$ : normalized step responses of the nonlinear model $(-)$ and approximate realization (•) (left figure); singular values of the Hankel matrix (right figure)
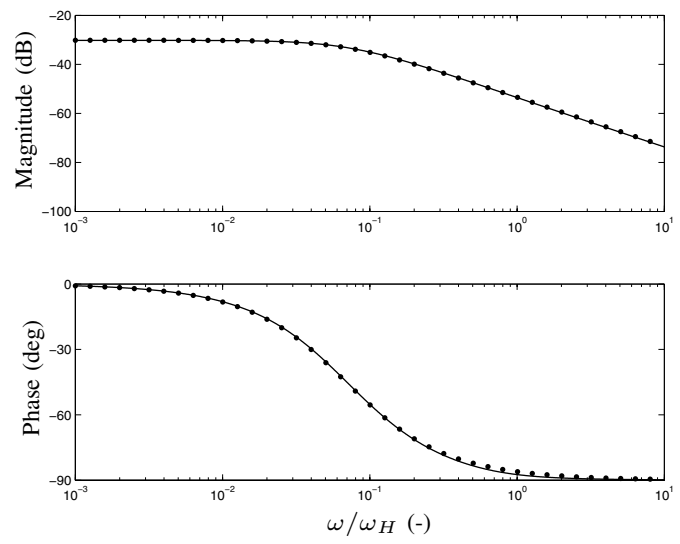

Fig. 8. Bode diagram for the linear compression system with valve dynamics (-) and the approximated realization (•): $N=9000 \mathrm{rpm}$, $B=1.07, \phi_{c 0}=2.5 \phi_{c, s}, \tilde{u}_{s}=0.01, n=2000$, and $f_{s}=1.024 \mathrm{kHz}$.

we constructed a compensator for the non-ideal valve dynamics. By filtering the measured step response with this compensator, an almost ideal step response signal could be obtained that was usable in the identification method. Of course, the construction of a compensator in practice requires accurate knowledge of the valve dynamics. If not available from the manufacturer, these data should be obtained from measurements.

Figure 7 shows the results from simulations with the more realistic compression system model. An approximation for the linearized system is shown in Fig. 8. The averaged estimation errors for various cases, obtained from 25 simulations each, were about $3-5 \%$. We also point out that the singular value plot in Fig. 7 justifies the use of a first order approximation. From the presented results we conclude that the proposed identification method is suitable to obtain an accurate estimate of the stability parameter $B$ for the actual compression system.

\section{SUMmARY AND CONCLUSION}

In this paper we have presented a dynamic model for an industrial centrifugal compressor test rig. Two nonlinear differential equations are derived for the compression system, analogous to the well-known Greitzer model. To account for the return piping in the system, an additional dimensionless parameter $F$ was introduced.

By comparing simulation results with surge measurements, we showed that both the amplitude and frequency of the surge oscillations were accurately predicted. However, the model parameters, in particular the important stability parameter $B$, were not uniquely defined when only predicted and measured surge cycles were matched. Different parameter values resulted in totally different local dynamic behavior, while the surge cycle predictions remained almost the same.

In this paper we proposed a solution for this problem, namely a straightforward identification method to determine the actual $B$ parameter. Based on step response data and an approximate realization algorithm, a value for $B$ could be determined. The proposed method makes use of the fact that the effective input-output behavior of the model can be captured by a first order dynamical system. With some extensions to make it applicable in practice, we performed simulations to show that the identification method can provide an estimate for the stability parameter in a centrifugal compression system.

Further work should now focus on applying the identification method to actual step response measurements of the setup. More detailed measurements should be performed in order to link the value of $B$ to the geometry of the compressor ducts and volumes.

\section{REFERENCES}

[1] E. M. Greitzer, "Surge and rotating stall in axial flow compressors: Part I-Theoretical compression system model," ASME Journal of Engineering for Power, vol. 98, pp. 190-198, 1976.

[2] J. T. Gravdahl, F. Willems, B. de Jager, and O. Egeland, "Modeling fo surge control of centrifugal compressors: comparison with experiment," in Proc. 39th IEEE Conference on Decision and Control, Sydney, Australia, 2000.

[3] F. P. T. Willems, "Modeling and bounded feedback stabilization of centrifugal compressor surge," Ph.D. dissertation, Eindhoven University of Technology, Eindhoven, The Netherlands, 2000.

[4] J. B. van Helmont, A. J. J. van der Weiden, and H. Anneveld, "Design of optimal controllers for a coal fired Benson boiler based on a modified approximate realization algorithm," Application of Multivariable System Techniques, pp. 313-320, 1990.

[5] F. K. Moore and E. M. Greitzer, "A theory of post-stall transient in axial compression systems: Part I-Development of equations," ASME Journal of Engineering for Gas Turbines and Power, vol. 108, no. 1, pp. 68-76, 1986.

[6] C. H. J. Meuleman, "Measurement and unsteady flow modelling of centrifugal compressor surge," Ph.D. dissertation, Eindhoven University of Technology, Eindhoven, The Netherlands, 2002.

[7] E. M. Greitzer, "Surge and rotating stall in axial flow compressors: Part II-Experimental results and comparison with theory," ASME Journal of Engineering for Power, vol. 98, pp. 199-217, 1976.

[8] J. S. Simon, L. Valavani, A. H. Epstein, and E. M. Greitzer, "Evaluation of approaches to active compressor surge stabilization," ASME Journal of Turbomachinery, vol. 115, pp. 57-67, 1993.

[9] B. de Schutter, "Minimal state-space realization in linear system theory: An overview," Journal of Computational and Applied Mathematics, vol. 121, pp. 331-354, 2000.

[10] S. Kung, "A new identification and model reduction algorithm via singular value decompositions," in Proc. 12th Asilomar Conference on Circuits, Systems, and Computers, Pacific Grove, CA, USA, 1978.

[11] R. J. Hesseling, "Active restraint systems, feedback control of occupant motion," Ph.D. dissertation, Eindhoven University of Technology, Eindhoven, The Netherlands, 2002. 